\title{
Integrating-sphere measurements for determining optical properties of tissue-engineered oral mucosa
}

\author{
A. M. Ionescu \\ anaionescu@ugr.es \\ J. C. Cardona
}

I. Garzón

A. C. Oliveira

\section{R. Ghinea}

\section{Alaminos}

\section{M. Pérez}

Department of Optics, Faculty of Sciences, University of Granada, Granada. ES-18071, Spain

Department of Optics, Faculty of Sciences, University of Granada, Granada. ES-18071, Spain

Department of Histology, Faculty of Medicine, University of Granada, Granada. ES- 18071, Spain

Department of Histology, Faculty of Medicine, University of Granada, Granada. ES- 18071, Spain

Department of Optics, Faculty of Sciences, University of Granada, Granada. ES-18071, Spain

Department of Histology, Faculty of Medicine, University of Granada, Granada. ES- 18071, Spain

Surgical procedures carried out in the oral and maxillofacial region can result in large tissue defects. Accounting for the shortage of oral mucosa to replace the excised tissues, different models of an organotypic substitute of the oral mucosa generated by tissue engineering have recently been proposed. In this work, the propagation of light radiation through artificial human oral mucosa substitutes based on fibrin-agarose scaffolds (fibrin, fibrin-0.1\% agarose, fibrin-0.2\%agarose) is investigated, and their optical properties are determined using the inverse adding-doubling (IAD) method based on integrating-sphere measurements. Similar values for the absorption and scattering coefficients between the fibrin and fibrin-0.1\% agarose bioengineered tissues and the native oral mucosa were found. These results suggest the adequacy of these biomaterials for potential clinical use in human oral mucosa applications. These optical properties represent useful references and data for applications requiring the knowledge of the light transport through this type of tissues, applications used in clinical practice. It also provides a new method of information analysis for the quality control of the development of the artificial nanostructured oral mucosa substitutes and its comparison with native oral mucosa tissues.

[DOI: http://dx.doi.org/10.2971/jeos.2015.15012]

Keywords: Human oral mucosa, scattering, absorption, tissue engineering

\section{INTRODUCTION}

The medical applications of optical radiation for diagnosis and treatment have progressively increased over the last years. In the field of dentistry, there is a growing awareness of the utility of various lasers in the modern dental practice where they can provide new alternatives and support to traditional approaches. Low power laser energy has found numerous uses in diagnosis, both in clinical settings and in dental research, such as confocal microscopic imaging of soft tissues, flow cytometric analysis of cells and cell sorting and profiling of tooth surfaces and dental restorations [1]. However, the optical imaging techniques have several limitations due to the strong light scattering in superficial tissues which can cause a decrease of spatial resolution and small penetration depth [2]. This fundamental optical property, together with the absorption has a strong influence on the distribution and propagation of photons in laser-irradiated tissues. Therefore, understanding the fundamental properties of tissues has become imperative in the development of imaging devices for oral diseases in the spectral range of interest, requiring a detailed knowledge of optical parameters to predict performance and effectiveness [3].

Laser applications have been shown to be of particular interest in oral soft tissues surgery due to the many advantages that it may offer compared to conventional instruments [4]. In particular, laser equipments can be easily used for surgical treatment of vascular lesions such as haemangioma and lymphangioma, thanks to the possibility to control bleeding and avoid the use of suture [5]. The degree of absorption in key tissue components indicates the type of effect gained by the laser on soft tissue, the content of water being important for the efficient absorption of many commonly used dental lasers [6].

However, large tissue defects can frequently result from different surgical procedures carried out in the oral and maxillofacial region [7]. Reconstruction of these defects represents a real challenge since maxillofacial surgeons are often confronted with the lack of oral mucosa to replace the excised tissues [8]. The oral mucosa acts as a barrier against the external environment. Loss of this barrier function causes dehydration 
and a high risk of infection. Reconstructions in the oral cavity, as required after tumor resections or cleft palate repair, are often complicated by similar problems. In the last two decades, the field of tissue engineering has provided new solutions to these problems. By using tissue engineering techniques, some researchers have recently proposed different models of an organotypic substitute of the oral mucosa [7]-[11].

Although some of the histological and morphological properties of tissue-engineered oral mucosa have been described [10, 12], to the best of our knowledge a systematic investigation of the propagation of light radiation through artificial human oral mucosa has not been performed. In this sense, lasers are used in several measuring devices as a coherent light source in order to properly determine different optical properties of biological tissues at specific wavelengths. The evaluation of these properties enhances the investigation of the behavior of transmitted light throughout the tissue and provides control of the adequacy of an artificial specimen in comparison with the native tissue to be replaced. The aim of the present work is to determine the optical properties of artificial human oral mucosa in the visible region, using the inverse adding doubling (IAD) method [13]. One integrating sphere is employed to measure the diffuse reflectance and diffuse transmittance for a new nanostructured model of artificial human oral mucosa and, also, for native rabbit oral mucosa, used as control sample.

The radiative transport equation has been widely used in order to determine the quantitative distribution of light intensity in biological media [3]. The correspondent calculations of light distribution require the knowledge of the absorption and scattering coefficients ( $\mu_{a}$ and $\mu_{s}$ respectively) and the anisotropy coefficient $(g)$. Therefore, an appropriate experimental method is necessary to measure these fundamental optical properties. Nevertheless, it is impossible to measure directly either the absorption or scattering in a single measurement. In order to solve such a difficult problem, different theoretical models as well as numerical or computational techniques have been developed. An important numerical approach known as Inverse Adding Doubling (IAD) [13] has been used to solve the radiative transport equation [14]. This method has provided accurate estimates of optical properties $\left(\mu_{a}, \mu_{s}\right.$ and $g$ ) of turbid media such as biological tissues [3], [15]-[18]. The entire process of IAD uses two dimensionless quantities, the albedo $(a)$ and the optical depth $(\tau)$, which are defined as:

$$
a=\frac{\mu_{s}}{\mu_{s}+\mu_{a}}
$$

and

$$
\tau=t\left(\mu_{s}+\mu_{a}\right)
$$

where $t$ is the physical thickness of the sample entered into the program in millimeters. Values of the refractive index $(n)$, anisotropy coefficient $(g)$, total diffuse reflectance $\left(R_{d}\right)$ and total diffuse transmittance $\left(T_{d}\right)$ of the samples are input to the IAD computer program which returns the values of the absorption coefficient $\left(\mu_{a}\right)$ and the reduce scattering coefficient $\left(\mu_{s}^{\prime}=\mu_{s}(1-g)\right)$. Further details of the IAD method can be found in the work of Prahl et al. [13]. According to Prahl et al. [13], since both anisotropy phase function and
Fresnel reflection at boundaries are accurately approximated, the IAD method is well suited to optical measurements for biological samples sandwiched between two glass slides.

\section{MATERHIALS AND METHODS}

\subsection{Human tissue samples}

Twenty small biopsies of oral mucosa were obtained from healthy donors undergoing minor oral surgery during local anesthesia at the School of Dental Sciences at the University of Granada, Spain. They corresponded to normal keratinized oral mucosa located at the retromolar trigonum. Immediately after extraction, all tissues were kept in a transport medium at $4{ }^{\circ} \mathrm{C}$ containing Dulbeccos Modified Eagles Medium (DMEM), $100 \mathrm{U} / \mathrm{ml}$ penicillin G, $100 \mu \mathrm{l} / \mathrm{ml}$ streptomycin and $0.25 \mu \mathrm{g} / \mathrm{ml}$ amphotericin $\mathrm{B}$, and processed before 24h. All patients provided informed consent to participate in the study and the study was approved by the local research committee.

\subsection{Construction of oral mucosa substitutes by tissue engineering}

Primary cultures of oral mucosa fibroblasts were generated as previously described $[10,11]$. Stromal samples were isolated from small fragments of oral mucosa, using collagenase I (Invitrogen-Gibco) at $37^{\circ} \mathrm{C}$ for $6 \mathrm{~h}$. Once the cells had been harvested by centrifugation, stromal fibroblasts were cultured in DMEM supplemented with 10\% fetal bovine serum (FBS, Sigma- Aldrich), $4 \mathrm{mM}$ L-glutamine, and 1\% antibioticantimycotic solution (Invitrogen-Gibco). All cells were incubated at $37^{\circ} \mathrm{C}$ in $5 \%$ carbon dioxide under standard culture conditions.

Then, 3 types of bioengineered oral mucosa stromal substitute were generated in the laboratory: human fibrin stromas with increasing concentration of agarose $(0 \%, 0.1 \%$ and $0.2 \%)$. In all cases, $21 \mathrm{ml}$ of human plasma were added to $1.8 \mathrm{ml}$ of DMEM in which 250,000 cultured fibroblasts had been previously suspended, and $200 \mu \mathrm{L}$ of tranexamic acid (Amchafibrin, FidesEcofarma, Valencia, Spain) were added to avoid spontaneous fibrinolysis. Fibrinolysis is a natural process that normally appears when human plasma coagulates, process that occurs in approximately eight days. During this time, the fibrinolytic agents contribute to the rupture of the clot. For these reason, in the development of artificial tissues based on fibrin biomaterials, the use of antifibrinolytic agents is a crucial step to avoid the fibrinolytic process in order to guarantee the three dimensional stability of the construct. As mentioned before, in our study, the antifibrinolytic agent used was tranexamic acid. This agent is a synthetic derivative of the amino acid lysine that acts by binding to plasminogen and blocking the interaction of plasminogen with fibrin, thereby preventing dissolution of the fibrin clot [19]. In this context, several research groups have reported the use of tranexamic acid as an inhibitor of the fibrinolytic agents [20]-[23]. After the addition of the tranexamic acid, $2 \mathrm{ml}$ of $1 \%$ of $\mathrm{CaCl} 2$ were added to the solution to precipitate the polymerization reaction of the hydrogel. In the case of the fibrinagarose gels, previously melted type VII agarose dissolved in PBS (phosphate-buffered saline) was supplemented in the last step. After polymeriza- 
tion, $15 \mathrm{ml}$ of culture medium were added to the surface of the bioengineered oral mucosa substitutes, which were incubated at $37^{\circ} \mathrm{C}$ in $5 \%$ carbon dioxide. Samples of the different oral mucosa substitutes were studied weekly until four weeks of development in culture.

Once generated before being subjected to the optical measurements all samples were nanostructured [24]. For nanostructuring, samples were transferred to a specific polycarbonate chamber in which 4-6 layers of sterile Whatman 3MM absorbent paper were put above and below the sample to facilitate dehydration of the artificial tissue. To prevent the oral mucosa stromal construct sticking to the paper layers, a filter nylon membrane $(0.4 \mu \mathrm{m}$ pore size $)$ was settled between both faces of the sample and the paper layers. Then, a flat crystal surface was set on top of the system and a total of $1000 \mathrm{~Pa}$ of pressure was applied to compress the bioengineered tissues. Previously, the authors research group successfully used this nanostructuring method to generate artificial human corneas [25] with physical properties similar to the native cornea. The nanostructuring method induces a series of complex interfibrillar changes at the nanometrical scale (nanostructuring) that modify the properties of this biomaterial.

This process was carried out for $3 \mathrm{~min}$, and then the nanostructured tissues were removed from the chamber and maintained in PBS until the moment of the analysis. Once generated, the sample thickness was determined using a Nikon Eclipse 90i light microscope. All bioengineered oral mucosas were generated and analyzed in triplicates. As controls, 3 fresh rabbit oral mucosa were obtained from adult rabbits immediately after death and were subjected to the same optical tests as the bioengineered artificial oral mucosa constructs.

\subsection{Histological analysis}

Histological analysis was performed by Hematoxilin-Eosin (H\&E), picrosirius, proliferating cell nuclear antigen (PCNA) and cortactin detection. For this, samples were fixed in $10 \%$ buffered formalin, dehydrated and embedded in paraffin. Then, sections about $5 \mu \mathrm{m}$ in thickness were cut in order to perform the whole histological analysis. First, H\&E analysis were carried out using standard protocol in order to determine the cell number by using Image J software (MacBiophotonics, Ontario, Canada), expressing all measurements as mean $\pm \mathrm{SD}$. Secondly, to determine the presence of collagen fibers, picrosirius staining was carried out using picrosirius staining solution for 40 minutes and counterstained with Harris hematoxilin. Finally, to evaluate cell proliferation and cell migration, PCNA and cortactin were detected by standard immunohistochemical. Briefly, paraffin was removed from sample sections using xylene, and endogenous peroxidase was quenched in 3\% $\mathrm{H} 2 \mathrm{O} 2$. Then, for antigen retrieval, we used citrate buffer ( $\mathrm{pH}$ 6.0) (Dako target retrievel solution) at $98^{\circ} \mathrm{C}$ for $40 \mathrm{~min}$. Incubation with the primary antibodies mouse anti-PCNA (1:1000 dilution) (Sigma p8825 clone PC10, USA) and rabbit anti-cortactin (1:100 dilution) (Abcam 1208 EP1922Y, USA) was performed at room temperature for $1 \mathrm{~h}$. Then, prediluted secondary antibodies (ImmPRESSReagent Kit, Vector, Burlingame, CA, USA)were used and the slides were developed using NovaRED substrate (Vector, Burlingame, CA, USA) for PCNA and 3,3'-diaminobenzidine DAB (Vector, Burlingame, CA, USA) for cortactin counterstained with Mayers hematoxilin.

For Picrosirius, anti-PCNA and anti-cortactin detection, a qualitative histological evaluation was carefully performed using a Nikon Eclipse 90i microscope.

\subsection{Determination of the optical properties}

Inverse adding doubling method was used to find the scattering and absorption of the artificial human oral mucosa and native oral mucosa using total reflection and total transmission measurements. A schematic of the experimental setup for reflection and transmission measurements needed for determining the total diffuse reflectance and total diffuse transmittance is shown in Figure 1. Neutral density filters were used to reduce the intensity of the incident lasers beams in order to prevent oversaturation of the detector. The detector (53-2754 Model, Coherent, USA) was attached to the integrating sphere and powered by a common power supply (C2719, Hamamatsu, Japan). The signal from the detector was measured by a digital multimeter (34401A Model, Agilent Technologies, USA).

Total diffuse reflection measurements were made using a $158.2 \mathrm{~mm}$-diameter integrating sphere (Oriel, model 70674. Newport. USA) with an $11 \mathrm{~mm}$-diameter detector port and a $15 \mathrm{~mm}$-diameter sample port with a baffle between ports. The entrance port had $15 \mathrm{~mm}$ in diameter. The measurements were performed on the nanostructured human oral mucosa and rabbit oral mucosa at $457.9 \mathrm{~nm}, 488 \mathrm{~nm}$ and $514.5 \mathrm{~nm}$ from an argon ion laser (Stellar-Pro-L Model, Modu-Laser, USA) and $632.8 \mathrm{~nm}$ from a He-Ne laser (30564 Model, Research ElectroOptics, USA). The maximum output power of lasers was 1000 $\mathrm{mW} \pm 5 \%$ for the argon laser and $12 \mathrm{~mW} \pm 0.2 \%$ for the HeNe laser. The diameter of both argon and He-Ne lasers beam was $2 \mathrm{~mm}$. Three reflection measurements were made on each sample (Figure 1(a)). Measurements were referenced to a $98 \%$ Optopolymer reflectance standard (OPST3-C, Optopolymer, Germany) (Figure 1(b)) and a dark measurement where the sample port was empty (Figure 1(c))). The tissue sample was placed between glass plates to prevent dehydration, to provide mechanical support and to minimize surface effects [26]. Its lateral size always exceeded the diameter of the respective sphere port.

The total diffuse reflectance, in terms of percentage, was calculated using

$$
R_{d \text { sample }}=r_{\text {std }} \frac{V R_{\text {sample }}-V R_{0}}{V R_{\text {std }}-V R_{0}}
$$

where $r_{s t d}$ is the reflection of the reference standard, $V R_{\text {sample }}$ is the reflection measurement of the sample, $V R_{s t d}$ is the reflectance standard measurement and $V R_{0}$ is the reflection measurement with the empty port (dark measurement).

Total diffuse transmission measurements were performed with the same integrating sphere using a mirror system 


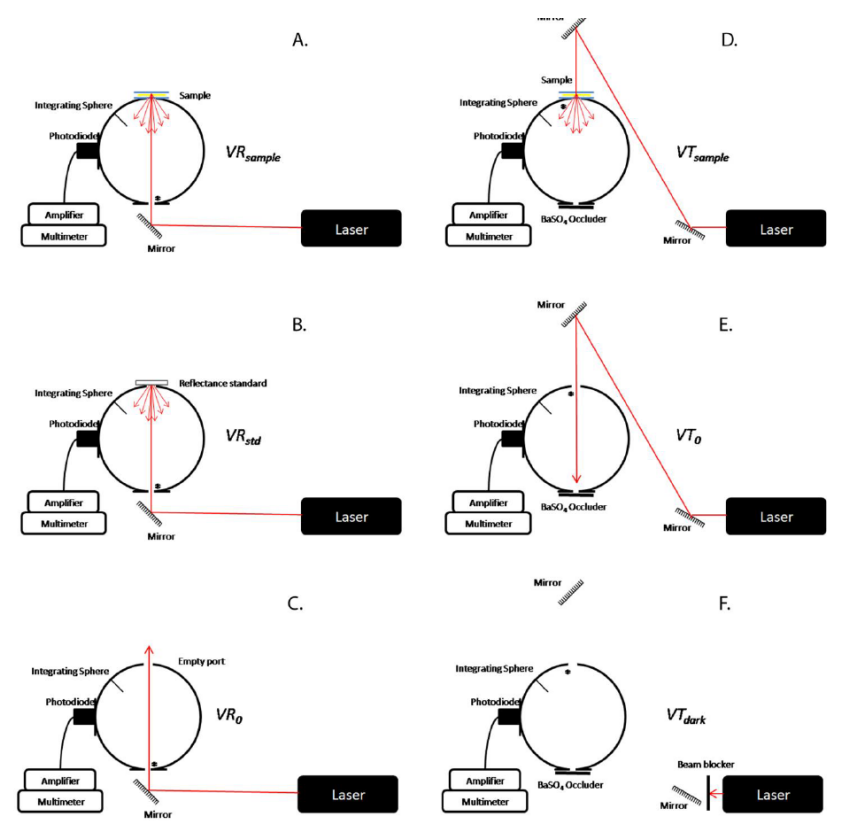

FIG. 1 Schematic representation of the experimental setup. A-C) Configuration for Reflection measurements; and D-F) Configuration for Transmission measurements.

* represents the entrance port for each measurement.

to divert the laser beam in order to not move the sample after the reflection measurements, so that the reflection and transmission measurement could be made in the same spot of the sample (Figure $1(\mathrm{~d})-(\mathrm{f})$ ). In this setup, only two ports were open, the $15 \mathrm{~mm}$-diameter sample port and the 11 mm-diameter detector port with a baffle between ports. Again, three transmission measurements were made on each tissue sample (Figure 1(d)). Measurements were referenced to $100 \%$ with the lasers illuminating the open port (empty port) (Figure 1(e)), and a dark measurement with an open port but with no illumination from the lasers (Figure 1(f)).

The total diffuse and transmittance, in terms of percentage, was calculated using

$$
T_{\text {d sample }}=\frac{V T_{\text {sample }}-V T_{\text {dark }}}{V T_{0}-V T_{\text {dark }}}
$$

where $V T_{\text {sample }}$ is the transmission measurement of the sample, $V T_{0}$ is the transmission measurement with the empty port (100\% reference measurement) and $V T_{\text {dark }}$ is the transmission measurement with no light entering the sphere, respectively.

Both types of measurements, reflectance and transmittance, were performed in a completely dark room. The only light source in the room during experiment was the laser source.

One critical parameter in both reflection and transmission measurements is the sphere wall reflectance. In order to determine this parameter, two measurements are needed (Figure 2). The integrating sphere was rotated with respect to the incident beam so that the light was directly incident upon the sphere wall between the sample port and the baffle. The reflectance of the sphere wall $r_{w}$ was calculated according to the following equation [13]

$$
\frac{1}{r_{w}}=a_{w}+a_{d} r_{d}\left(1-a_{\epsilon}\right)+a_{s} r_{s t d}\left(1-a_{s}\right) \frac{V R_{s t d}^{\text {diff }}}{V R_{s t d}^{\text {diff }}-V R_{0}^{\text {diff }}}
$$

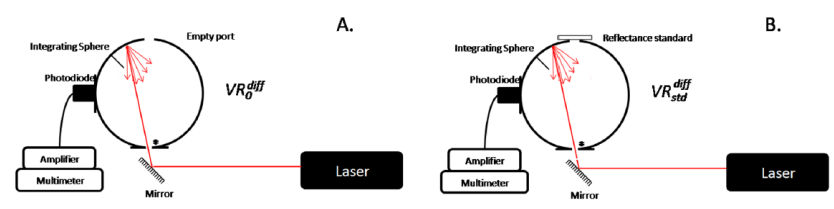

FIG. 2 Schematic representation of the experimental setup used for the reference sphere calibration measurements. * marks the entrance port of the sphere.

where $a_{w}$ is the fractional sphere wall area, $a_{d}$ the fractional detector area, $a_{s}$ the fractional sample area, $a_{e}$ the fractional entrance port area, $r_{d}$ is the detector reflectance and $r_{s t d}$ is the reflectance of the reflectance standard.

In addition, the thickness of each tissue sample was measured using a Nikon Eclipse 90i light microscope (Nikon Instruments Inc., USA) and also, the refractive index of the artificial oral mucosa samples was determined using an Abbe refractometer at $589 \mathrm{~nm}$ (PCE Iberica, Spain). Changes in the refractive index over the wavelengths used in the present study were assumed negligible.

All these parameters, together with the six additional measurements (three reflection measurements $-V R_{\text {sample }}, V R_{\text {std }}$, $V R_{0}$ - and three transmission measurements $-V T_{\text {sample }}, V T_{0}$, $V T_{\text {dark }^{-}}$) form a complete set of data, for each sample, necessary to determine its optical properties, for each of the wavelengths $457.9 \mathrm{~nm}, 488 \mathrm{~nm}, 514.5 \mathrm{~nm}$ and $632.8 \mathrm{~nm}$, using the inverse adding-doubling method developed by Prahl et al. [13]. This method has been widely used in tissue optics for processing experimental data obtained using integrating spheres $[15,16,27,28]$ since it offers the possibility to rapidly determine iterative solutions with the help of microcomputers.

Prior to the oral mucosa sample measurements, a calibration of the experimental setup was carried out using three polyurethane optical phantoms [29] with known optical properties. The maximum error obtained was $2.19 \%$ for the scattering coefficient and $4.65 \%$ for the absorption coefficient. These errors were taking into account when presenting and discussing the results.

\subsection{Statistical analysis}

As normality (and homogeneity of variance) assumptions were not satisfied, non-parametric tests were used. To test the significance of observed differences between the study groups, the Kruskal-Wallis one-way analysis of variance by ranks $(\mathrm{K}-\mathrm{W})$ and the Mann-Whitney $\mathrm{U}$ test $(\mathrm{M}-\mathrm{W})$ were applied. Considering the Bonferronis correction, a value of $p \leq 0.001$ was considered to be statistically significant.

\section{RESULTS and DISCUSSION}

Construction of an efficient artificial oral mucosa by tissue engineering represents a major scientific challenge. The use of these kinds of tissue substitutes can help oral and maxillofacial surgeons to improve the outcome of pa- 


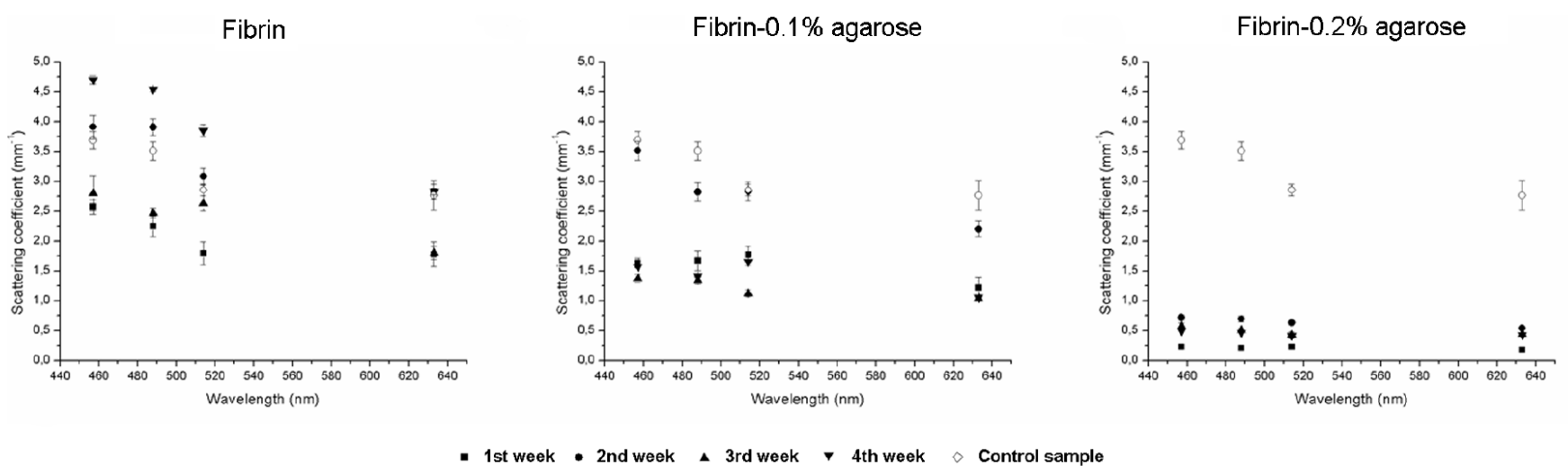

FIG. 3 Reduced scattering coefficient of the control and bioengineered oral mucosa samples during the four weeks of development in culture.

tients in need of large reconstructions involving the oral cavity $[9,11,23],[30]-[32]$. In this study, three types of artificial human oral mucosa substitutes (fibrin, fibrin with $0.1 \%$ and $0.2 \%$ agarose concentration) were successfully generated in the laboratory and could be used as in vitro models for investigations of experimental pharmacology or toxicology tests, thus avoiding the need for animal research [10, 33]. Previous results by Sanchez-Quevedo et al. [10] on the histological and histochemical evaluation of the human oral mucosa constructs developed by tissue-engineering, using the same method for the generation of the constructs as the one presented in the present work, proved that the fibrinagarose complexes satisfy the criteria for biomaterials used in tissue engineering of the human oral mucosa. Nevertheless, the novel technique of nanostructuring proposed by the authors of the present study and previously used for the successful development of human bioengineered corneas [25], contributes to provide useful solutions for the generation of artificial oral tissues by tissue engineering, since reconstructions in the oral cavity, as required after tumor resections, are often complicated. Moreover, Haugh et al. [34] showed that the plastic compression (concept the nanostructuring technique is based on) significantly increases the compressive properties of fibrin gels without impairing cellular viability and subsequent extracellular matrix synthesis.

Nowadays, laser-assisted procedures complement conventional treatment in many cases of oral disorders; in some areas laser treatment being now considered the therapy of choice. When applying these therapies, the precise knowledge of the optical properties of examined tissue and also of the surrounding tissues, acquires great importance. For this, the IAD algorithm provides an accurate estimation of the optical properties for biological tissues [15]-[18] from measurements of the index of refraction, scattering anisotropy coefficient, diffuse total reflectance and diffuse total transmittance.

In this work, the measured refractive index values of the bioengineered oral mucosa substitutes varied from 1.330 to 1.340. For each sample, measurements were repeated three times and the obtained values agreed to within $5 \%$. For all of the IAD calculations, an average value of 1.335 was assumed for all samples analyzed. Since only measurements of the diffuse reflection and transmission were made, the average scattering anisotropy coefficient of both artificial and control oral mu- cosa tissues was assumed to be 0.9 , value in the range of the anisotropy coefficient for biological tissues [3, 18], [35]-[38].

The thickness of the sample was measured weekly, after the nanostructuration process and before the performance of the optical measurements. The thickness values varied between 0.3 and $1 \mathrm{~mm}$.

The total diffuse reflectance $\left(R_{d}\right)$ and total diffuse transmittance $\left(T_{d}\right)$ measured at $457.9 \mathrm{~nm}, 488 \mathrm{~nm}, 514.5 \mathrm{~nm}$ from the argon laser and $632.8 \mathrm{~nm}$ from the He-Ne laser, along with the values of the thickness, refractive index and scattering anisotropy coefficient, were input into the IAD program. The output of the IAD program consisted on the reduced scattering $\left(\mu_{s}^{\prime}\right)$ and absorption $\left(\mu_{a}\right)$ coefficients.

Figure 3 displays the spectral behavior of the reduced scattering coefficient of the control and bioengineered oral mucosa samples during the four weeks of development in culture. The values shown in Figure 3 represent the mean values of the reduced scattering coefficients of the oral mucosa contructs, after taking into account the error values obtained during the calibration of the experimental setup $(2.19 \%$ for the scattering coefficient and $4.65 \%$ for the absorption coefficient). The highest values of reduced scattering coefficient were obtained for the fibrin oral mucosa sample, followed by the fibrin- $0.1 \%$ agarose and fibrin- $0.2 \%$ agarose samples, respectively. In general, as expected for biological tissues, the reduced scattering decreased with increasing wavelength. The time of maturation in culture played an important role in the development of the scattering properties of the bioengineered samples. The scattering properties of the fibrin and fibrin with $0.1 \%$ agarose artificial tissues increased until the second week in culture, decreased for the third week, to increase again in the fourth week in culture. This increase is more pronounced for the fibrin sample, which showed the highest reduced scattering coefficient values after four weeks of development in culture, than for the fibrin- $0.1 \%$ agarose sample, which showed the highest reduced scattering coefficient values after only two weeks of development in culture. Nevertheless, these maximum values of the reduced scattering for both fibrin and fibrin with $0.1 \%$ agarose bioengineered samples were similar to that of the control oral mucosa, with no statistically significant differences in the second week of development in culture both for fibrin $(p=0.101)$ and fibrin with $0.1 \%$-agarose $(p=0.026)$. 


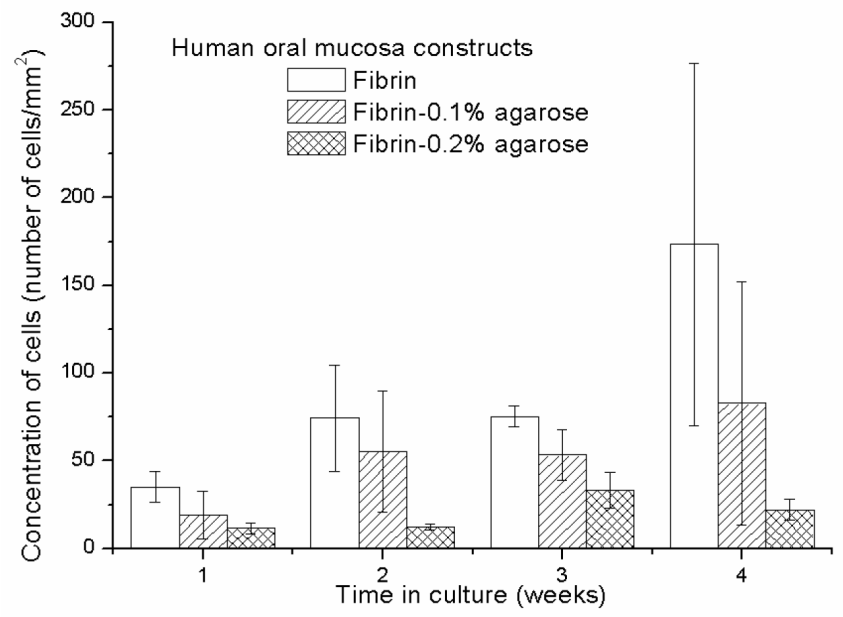

FIG. 4 Concentration of cells of the three types of nanostructured oral mucosa as a function of time of development in culture.

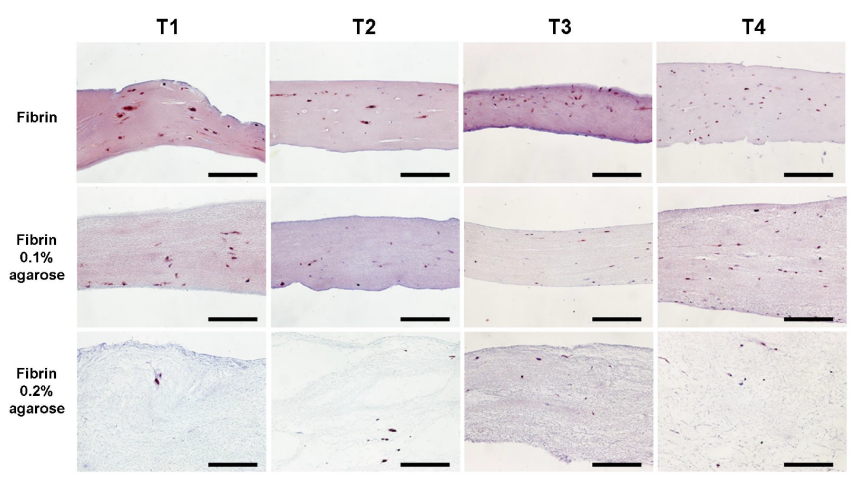

FIG. 5 Immunohistochemical analysis of PCNA in artificial oral mucosa stromas during the four weeks in culture. Scale bar $=200 \mu \mathrm{m}$

Mourant et al. $[35,38]$ found that, for suspensions of biological cells in PBS, the main candidates for scattering centers in mammalian cells are the cell itself, the nucleus and other cellular organelles, the shape of the cell having very little effect on the scattering properties of the tissue. In our case, when analyzing the number of cells of the bioengineered substitutes, it was found that the fibrin substitutes displayed higher concentration of cells than the fibrin-agarose oral mucosa substitutes during the entire period of time in culture (Figure 4), as well as higher scattering values. However, it should be stated that this behavior is very likely to be tissue dependent, therefore other tissues might display different scattering behavior in relation to the cell number. Our results are in agreement with the findings of San Martin et al. [23] who revealed a complete degradation of the most of the fibrin in the fibrin constructs after four weeks of development in culture, whereas the fibrin degradation was much slower in the fibrin- $0.1 \%$ agarose constructs.

The scattering properties of the fibrin with $0.2 \%$ agarose concentration were the lowest of all samples, with maximum values after 2 weeks of development in culture. However, these maximum values reached only $20 \%$ of the values of the control rabbit oral mucosa tissue. This result suggests that the amount of agarose in the artificial human oral mucosa constructs plays an important role in the development of their optical properties. Moreover, the histological analysis showed that the cells

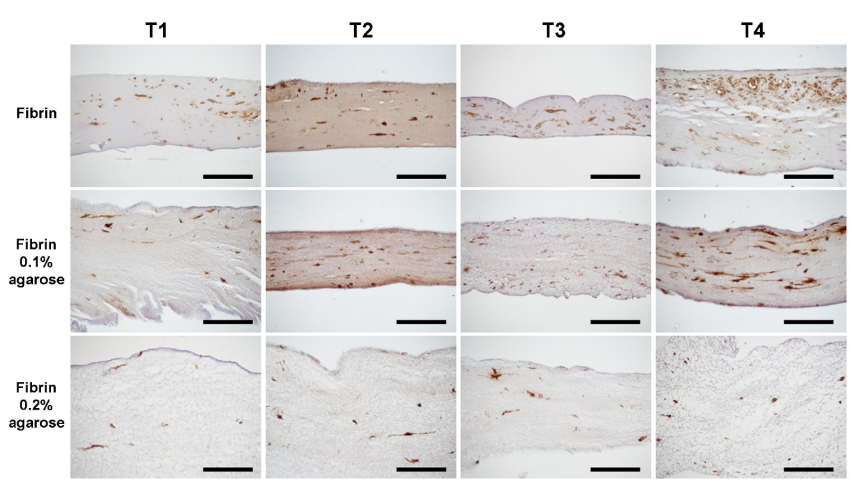

FIG. 6 Immunohistochemical analysis of cortactin in artificial oral mucosa stromas during the four weeks in culture. Scale bar $=200 \mu \mathrm{m}$

proliferation and migration are considerably influenced by the amount of agarose present in the bioengineered constructs. In the fibrin-agarose scaffolds, the proliferation and migration rates of the fibroblasts were lower when compared to the fibrin scaffolds, especially in the fibrin with $0.2 \%$ agarose concentration as revelead by the PCNA (Figure 5) and cortactin analysis (Figure 6), during the entire time of development in culture. However, these low values in the fibrin-agarose construct are due to the small number of cells present in this type of scaffold, especially for the one with $0.2 \%$ agarose.

Agarose type VII-A is a linear polysaccharide hydrogel extracted from marine red algae. It undergoes polymerization and solidifies in response to low temperatures (the term low refers to a $26{ }^{\circ} \mathrm{C} \pm 2{ }^{\circ} \mathrm{C}$ temperature that can be considered as low compared to the gel point of routinely used agarose 50$55^{\circ} \mathrm{C}$ ), thereby providing a 3D environment for the cells [39]. However, agarose is not commonly used in tissue engineering due to the reduced growth rate of cells grown on these hydrogels [40]. This behavior was confirmed in the current study in which cell growth and the ability to migrate were reduced in the bioengineered scaffolds with small amounts of agarose. Despite this, the mechanical behavior of fibrin-agarose scaffolds has been demonstrated to improve, and therefore the stability of the constructs has increased when agarose is used for generating bioengineered substitutes [25, 41]. These two attributes are important in facilitating surgical implant procedures [23].

Interestingly, the collagen expression was detectable by immunohistochemistry in all three types of substitutes, although in the fibrin with $0.2 \%$ agarose constructs this expression was delayed. This might be due to the amount of agarose present in the oral mucosa constructs.

Collagen fibrillogenesis is a multistep process that is precisely regulated and involves collagen assembly to form fibrils intermediates and assembly of these to form mature fibrils $[42,43]$. Thus, the samples of fibrin and fibrin $0.1 \%$ agarose suffer a remodeling of the collagen fibrils after 2 two weeks of development (Figure 7) that could explain also the variation in the scattering coefficient values, since it has been well established that the spatial arrangement of the collagen fibrils, the variation of their diameters and the number density have a strong 


\begin{tabular}{|c|cccc|c|}
\hline \multicolumn{7}{|c|}{ Absorption coefficient $\left(\mathrm{mm}^{-1}\right)$} \\
\hline \multirow{2}{*}{ Wavelength (nm) } & \multicolumn{7}{|c|}{ Fibrin oral mucosa sample } & \multirow{2}{*}{ Native rabbit } \\
& $\mathrm{T} 1$ & $\mathrm{~T} 2$ & $\mathrm{~T} 3$ & $\mathrm{~T} 4$ & \\
\hline 457.9 & 0.063 & 0.068 & 0.048 & 0.147 & 0.237 \\
4880 & 0.069 & 0.100 & 0.005 & 0.177 & 0.225 \\
514.5 & 0.014 & 0.000 & 0.185 & 0.024 & 0.159 \\
632.8 & 0.000 & 0.088 & 0.000 & 0.081 & 0.238 \\
\hline \multirow{2}{*}{ Wavelength (nm) } & Fibrin-0.1\% agarose oral mucosa sample & \multirow{2}{*}{ Native rabbit } \\
& T1 & T2 & T3 & T4 & \\
\hline 457.9 & 0.012 & 0.175 & 0.000 & 0.028 & 0.237 \\
488 & 0.006 & 0.054 & 0.000 & 0.032 & 0.225 \\
514.5 & 0.137 & 0.038 & 0.000 & 0.023 & 0.159 \\
632.8 & 0.014 & 0.029 & 0.000 & 0.040 & 0.238 \\
\hline \multirow{2}{*}{ Wavelength (nm) } & Fibrin-0.2\% agarose oral mucosa sample & \multirow{2}{*}{ Native rabbit } \\
& T1 & T2 & T3 & T4 & \\
\hline 457.9 & 0.003 & 0.009 & 0.000 & 0.003 & 0.237 \\
4880 & 0.000 & 0.009 & 0.000 & 0.010 & 0.225 \\
514.5 & 0.000 & 0.000 & 0.046 & 0.000 & 0.159 \\
632.8 & 0.000 & 0.012 & 0.044 & 0.012 & 0.238 \\
\hline
\end{tabular}

TABLE 1 Absorption coefficient values of the oral mucosa substitutes as calculated using the IAD program.

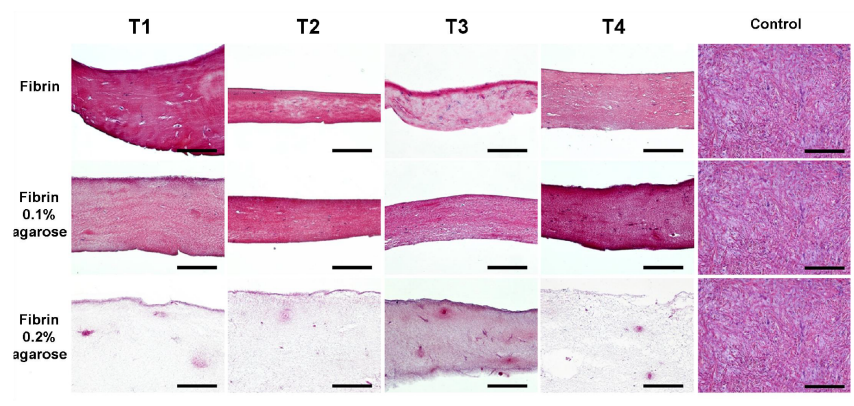

FIG. 7 Immunohistochemical analysis of collagen type I in control and artificial oral mucosa stromas stained with Picrosirius. Scale bar $=200 \mu \mathrm{m}$

influence on the light scattering throughout the biological tissues [44].

All the artificial constructs of human oral mucosa displayed small or no absorption properties (Table 1) whose values showed variation across the wavelengths studied. Statistically significant differences were found when comparing the absorption values of the artificial constructs at all times of development in culture with the ones of the control rabbit oral mucosa sample (K-W and M-W $p<0.001)$. In biological tissue the responsible cellular components for the absorption are proteins and nucleic acids. As mentioned before, the cells in the bioengineered oral mucosas generated in this study were able to synthesize collagen and PCNA, proving their mitotic activity and the presence of nucleic acids. However, highly complex structural proteins such as decorin and biglycan require more time to be synthesized and are absent in these samples and thus the low absorption values obtained during the period of time studied in this work.

The fibrin with $0.2 \%$ agarose concentration exhibited the lowest or no absorption properties, during all times of development in culture. This result could be explained by the fact that the synthesis and assembly of structural proteins requires a high number of synchronized cells that intercommunicate. A small number of cells, such as the case of fibrin-0.2\%agarose construct, cannot secrete an important amount of absorbing components in order to increase the absorption properties of this oral mucosa substitute. The results regarding the optical properties of the nanostructured oral mucosa artificial substitutes determined in the present study complete a comprehensive analysis on the morphological, histochemical and physical characterization of artificial human oral mucosa constructs generated by tissue engineering and based on fibrin and fibrin-agarose. Previous studies by Rodriguez et al. [41] proved that the addition to fibrin of a small amount of agarose allows the rheological stability of the oral mucosa substitute to be maintained. Moreover, San Martin et al. [23] show that these type of bioengineered tissues present histological and molecular similarities with native human oral mucosa stroma. In addition, they observed that the nature of the biomaterial behavior influenced the oral stromal fibroblasts, thereby modulating their growth, protein synthesis, and collagen fibrillogenesis. This feature, together with its viscoelastic and optical similitude with native tissues, make the fibrin and fibrin- $0.1 \%$ agarose bioengineered tissues appropriate for clinical use in human oral mucosa applications.

\section{CONCLUSIONS}

The actual values of the absorption and scattering coefficients for the artificial and native tissues reported in the present study provide useful references and data for applications requiring the knowledge of the light transport through this type of tissues, applications used in clinical practice. The results of this work suggest that the optical parameters of biological tissues in the diffuse theory model can be determined using the measuring technology of light radiation, and the optical parameters of bioengineered tissues and native tissues can be compared and analyzed. In this context, the regenerative medicine requires information about the relationship be- 
tween optical and biological properties of tissues, relationship that can provide important information about the physiological condition of the tissue. The determination of optical parameters could influence and control the development of new dental bioengineered tissues by comparing experimental tissues with native ones and determining this way, their adequacy for clinical applications.

\section{ACKN OWLEDGMENTS}

The authors acknowledge funding support from the research projects JA TEP-1136 from Junta de Andalucía, Spain and MAT2013-43946R from the Spanish Ministry of Economy and Competitiveness. . The authors would like to thank prof. dr. Scott Prahl from the Oregon Institute of Technology, USA, for providing the optical phantoms used for the calibration of the experimental setup used in this work.

\section{References}

[1] L. J. Walsh, "The current status of laser applications in dentistry," Aust. Dent. J. 48, 146-155 (2003).

[2] E. A. Genina, A. N. Bashkatov, and V. V. Tuchin, "Optical clearing in cranial bone," Adv. 0pt. Tech. 2008, 267867 (2008)

[3] D. K. Sardar, G. Y. Swanland, R. M. Yow, R. J. Thomas, and A. T. Tsin, "Optical properties of ocular tissues in the near infrared region," Lasers Med. Sci. 22, 46-52 (2007).

[4] M. Sulieman, "An overview of the use of lasers in general dental practice: 2. Laser wavelengths, soft and hard tissue clinical applications," Dent. Update 32, 286-288, 291-296 (2005).

[5] H. Deppe, and H. H. Horch, "Laser applications in oral surgery and implant dentistry," Lasers Med. Sci. 22, 217-221 (2007).

[6] L. J. Walsh, “Dental lasers: Some basic principles," Postgrad. Dent. 4, 26-29 (1994).

[7] S. Schultze-Mosgau, B. K. Lee, J. Ries, K. Amann, and J. Wiltfang, "In vitro cultured autologous pre-confluent oral keratinocytes for experimental prefabrication of oral mucosa," Int. J. Oral. Max. Surg. 33, 476-485 (2004).

[8] J. Song, K. Izumi, T. Lanigan, and S. E. Feinberg,"Development and characterization of a canine oral mucosa equivalent in a serum free environment," J. Biomed. Mater. Res. 71, 143-153 (2004).

[9] C. Lauer, and R. Schimming, "Tissue-engineered mucosa graft for reconstruction of the intraoral lining after freeing of the tongue: a clinical and immunohistologic study," Int. J. Oral. Max. Surg. 59, 169-175 (2001).

[10] M. C. Sanchez-Quevedo, M. Alaminos, L. M. Capitan, G. Moreu, I. Garzon, P. V. Crespo, and A. Campos, "Histological and histochemical evaluation of human oral mucosa constructs developed by tissue engineering," Histol. Histopathol. 22, 631-640 (2007).

[11] I. Garzón, M. C. Sánchez-Quevedo, C. Moreu, M. González-Jaranay, M. González-Andrades, A. Montalvo, M. Alaminos, and A. Campos, "In vitro and in vivo cytokeratin patterns of expression in bioengineered human periodontal mucosa," J. Periodontal Res. 44, 588-597 (2009).

[12] M. Moharamzadeh, I. M. Brook, R. Van Noort, A. M. Scutt, and M. H. Thornhill, "Tissue-engineered oral mucosa: a review of the scientific literature," J. Dent. Res. 86, 115-124 (2007).
[13] S. A. Prahl, M. J. C. Van Gemert, and A. J. Welch, “Determining the optical properties of turbid media by using the adding-doubling method," Appl. Optics 32, 559-568 (1993).

[14] S. Chandrasekhar, Radiative transfer (Ed. Dover, New York, 1960).

[15] D. K. Sardar, B. G. Yust, F. J. Barrera, L. C. Mimun, and A. T. C. Tsin, "Optical absorption and scattering of bovine cornea, lens and retina in the visible region," Lasers Med. Sci. 24, 839-847 (2009).

[16] A. N. Bashkatov, E. A. Genina, V. I. Kochubey, and V. V. Tuchin, "Optical properties of human skin, subcutaneous and mucous tissues in the wavelength range from 400 to 2000nm," J. Phys. D. Appl. Phys. 38, 2543-2555 (2005).

[17] D. K. Sardar, and L. B. Levy. "Optical properties of whole blood," Lasers Med. Sci. 13, 106-111 (1998).

[18] W. F. Cheong, S. A. Prahl, and A. J. Welch, "A review of the optical properties of biological tissues," IEEE J. Quantum Elect. 26, 2166-2171 (1990).

[19] P. L. McCormack, "Tranexamic acid: a review of its use in the treatment of hyperfibrinolysis," Drugs 72, 585-617 (2012).

[20] S. Llames, E. García, V. García, M. del Río, F. Larcher, J. L. Jorcano, E. López, et al., "Clinical results of an autologous engineered skin," Cell Tissue Bank 7, 47-53 (2006).

[21] I. Peõa, L. M. Junquera, A. Meana, E. García, V. García, and J. C. De Vicente, "In vitro engineering of complete autologous oral mucosa equivalents: characterization of a novel scaffold," J. Periodontal. Res. 45, 375-380 (2010).

[22] I Garzon, D. Serrato, 0. Roda, M. Del Carmen Sanchez-Quevedo, M. Gonzales-Jaranay, G. Moreu, R. Nieto-Aguilar, et al., "In vitro cytokeratin expression profiling of human oral mucosa substitutes developed by tissue engineering," Int. J. Artif. Organs 32, 711-719 (2009).

[23] S. San Martin, M. Alaminos, T. M. Zorn, M. C. Sánchez-Quevedo, I. Garzón, I. A. Rodriguez, and A. Campos, "The effects of fibrin and fibrin-agarose on the extracellular matrix profile of bioengineered oral mucosa" J. Tissue Eng. Regen. M. 7, 10-19 (2013).

[24] E. Hadjipanayi, M. Ananta, M. Binkowski, I. Streeter, Z. Lu, Z. F. Cui, R. A. Brown, et al., "Mechanisms of structure generation during plastic compression on nanofibrillar collagen hydrogel scaffolds: towards engineering of collagen," J. Tissue Eng. Reg. M. 5, 505-519 (2011).

[25] A. M. Ionescu, M. Alaminos, J. de la Cruz Cardona, J. de Dios GarcíaLópez Durán, M. González-Andrades, R. Chinea, A. Campos, et al., "Investigating a novel nanostructured fibrin-agarose biomaterial for human cornea tissue engineering: rheological properties," J. Mech. Behav. Biomed. 4, 1963-1973 (2011).

[26] J. W. Pickering, J. C. M. Moes, S. A. Prahl, and M. J. C. van Gemert, "Two integrating spheres with an intervening scattering sample," J. Opt. Soc. Am. A 9, 621-631 (1992).

[27] D. K. Sardar, R. M. Yow, A. T. Tsin, and R. Sardar, “Optical scattering, absorption and polarization of healthy and neovascularized human retinal tissues," J. Biomed. Opt. 10, 051501.1-8 (2005).

[28] B. G. Yust, L. C. Mimun, and D. K. Sardar, "Optical absorption and scattering of bovine cornea, lens, and retina in the near-infrared region," Lasers Med. Sci. 27, 413-422 (2012).

[29] T. Moffit, Y. C. Chen, and S. A. Prahl, "Preparation and characterization of polyurethane optical phantoms," J. Biomed. Optics 11, 041103.1-10 (2006).

[30] M. Ueda, K. Ebata, and T. Kaneda, "In Vitro fabrication of bioartificial mucosa for reconstruction of oral mucosa: Basic research and clinical application," Ann. Plast. Surg. 27, 540-549 (1991). 
[31] I. Schlenz, K. J. Korak, R. Kunstfeld, K. Vinzenz, H. Plenk Jr, and J. Holle, "The dermis-prelaminated scapula flap for reconstructions of the hard palate and the alveolar ridge: a clinical and histologic evaluation," Plast. Reconstr. Surg. 108, 1519-1524 (2001).

[32] K. Izumi, S. E. Feinberg, A. lida, and M. Yoshizawa "Intraoral grafting of an ex vivo produced oral mucosa equivalent: a preliminary report," Int. J. Oral. Max. Surg. 32, 188-197 (2003).

[33] M. Alaminos, M. Del Carmen Sánchez-Quevedo, J. I. Muõoz-Avila, D. Serrano, S. Medialdea, I. Carreras, and A. Campos, "Construction of a complete rabbit cornea substitute using a fibrin-agarose scaffold," Invest. Ophth. Vis. Sci. 47, 3311-3317 (2006).

[34] M. G. Haugh, S. D. Thorpe, T. Vinardell, C. T. Buckley, and D. J. Kelly, "The application of plastic compression to modulate fibrin hydrogel mechanical properties," J. Mech. Behav. Biomed. 16, 66-72 (2012).

[35] J. R. Mourant, J. P. Freyer, A. H. Hielscher, A. A. Eick, D. Shen, and T. M. Johnson "Mechanisms of light scattering from biological cells relevant to noninvasive optical-tissue diagnostics," Appl. Optics 37, 3586-3593 (1998).

[36] A. K. Popp, M. T. Valentine, P. D. Kaplan, and D. A. Weitz "Microscopic origin of light scattering in tissue," Appl. Opt. 42, 2871-2880 (2003).

[37] E. Salomatina, B. Jiang, J. Novak, and A. N. Yaroslavsky "Optical properties of normal and cancerous human skin in the visible and near-infrared spectral range," J. Biomed. Opt. 11, 064026.1-064026.9 (2006)
[38] J. R. Mourant, M. Canpolat, C. Brocker, 0. Esponda-Ramos, T. M. Johnson, A. Matanock, K. Stetter, et al., "Light scattering from cells: the contributions of the nucleus and the effects of proliferative status," J. Biomed. Opt. 5, 131-137 (2000).

[39] C. T. Buckley, S. D. Thorpe, F. J. O’Brien, A. J. Robinson, and D. J. Kelly, "The effect of concentration, thermal history and cell seeding density on the initial mechanical properties of agarose hydrogels," J. Mech. Behav. Biomed. 2, 512-521 (2009).

[40] A. C. Aufderheide, and K. A. Athanasiou, "Comparison of scaffolds and culture conditions for tissue engineering of the knee meniscus," Tissue Eng. 11, 1095-1104 (2005).

[41] I. A. Rodríguez, M. T. López-López, A. C. Oliveira, M. C. SánchezQuevedo, A. Campos, M. Alaminos, and J. D. Durán, "Rheological characterization of human fibrin and fibrin-agarose oral mucosa substitutes generated by tissue engineering," J. Tissue Eng. Regen. M. 6, 636-644 (2012).

[42] D. E. Birk, E. I. Zycband, and S. Woodruff, "Collagen fibrinogenesis in situ: fibril segments become long fibrils as the developing tendon matures," Dev. Dynam. 208, 291-298 (1997).

[43] T. Starborg, Y. Lu, K. E. Kadler, and D. F. Holmes "Electron microscopy of collagen fibril structure in vitro and in vivo, including three-dimensional reconstruction," Methods Cell. Biol. 88, 319-345 (2008).

[44] K. M. Meek, D. W. Leonard, C. J. Connon, S. Dennis, and S. Khan, "Transparency, swelling and scarring in the corneal stroma," Eye 17, 927-936 (2003). 